\title{
Genética molecular de caracteres cuantitativos en cruzamientos dialélicos de tomate
}

\author{
Guillermo Raúl Pratta(1), Sabina Lara Mahuad(1), Ariana Díaz(2), Ezequiel Marchionni Basté(1), \\ David Rodolfo Liberatti ${ }^{(1)}$, Gustavo Rubén Rodríguez ${ }^{(1)}$, Roxana Zorzoli( ${ }^{(1)}$
}

(1)Universidad Nacional de Rosario, Facultad de Ciencias Agrarias, Cátedra de Genética, Casilla de Correo no 14, S2125ZAA, Zavalla, Santa Fe, Argentina. E-mail: gpratta@unr.edu.ar, smahuad@unr.edu.ar, emarchio@unr.edu.ar, dliberat@unr.edu.ar, grodrig@unr.edu.ar, rzorzoli@unr.edu.ar ${ }^{(2)}$ Universidad de Morón, Facultad de Ciencias Exactas, Químicas y Naturales, Cabildo 134, B1708JPD, Morón, Buenos Aires, Argentina. E-mail: nanudiaz@hotmail.com

\begin{abstract}
Resumen - El objetivo de este trabajo fue evaluar marcadores moleculares y caracteres cuantitativos en un cruzamiento dialélico completo sin recíprocos, entre cinco líneas recombinantes de tomate y sus híbridos. Se obtuvieron perfiles de AFLP ("amplified fragment length polymorphism") y de polipéptidos del pericarpio en cuatro estados de madurez del fruto de 15 genotipos. Se evaluaron, entre otros: peso, acidez titulable, $\mathrm{pH}$, vida poscosecha y firmeza. Se calculó el porcentaje de polimorfismo para los marcadores moleculares y el porcentaje de variabilidad genética para los caracteres cuantitativos en el grupo de líneas recombinantes, el de híbridos y el conjunto de genotipos. Se realizaron análisis de agrupamiento con cada nivel de variación genética. Para AFLP, el porcentaje de polimorfismo varió entre 34 y $54 \%$ y, para los perfiles polipeptídicos, entre 40 y $78 \%$. Mayor polimorfismo fue observado en el grupo de híbridos. La variabilidad genética fue de $100 \%$ para acidez y $34 \%$ para firmeza, con los mayores valores en los parentales. La similitud genética varió entre los genotipos según el nivel de variación genética; pero la consistencia en el agrupamiento de algunas líneas recombinantes y sus híbridos fue conservada, lo que evidenció asociaciones entre los datos moleculares y fenotípicos.
\end{abstract}

Términos para indexación: Lycopersicon, biotecnología, calidad del fruto, mejoramiento genético vegetal, recursos fitogenéticos.

\section{Molecular genetics of quantitative traits in tomato diallel crosses}

\begin{abstract}
The purpose of this research was to evaluate molecular markers and some quantitative fruit traits, in a complete diallel cross without reciprocals, among five tomato recombinant inbred lines and their hybrids. Amplified fragment length polymorphism (AFLP) and pericarp polypeptide profiles were obtained in four stages of fruit maturity of 15 genotypes. Among other traits, fruit weight, titratable acidity, $\mathrm{pH}$, shelf life, and firmness were evaluated. The percentages of polymorphism for molecular markers and for the genetic variability for quantitative characters were calculated in the recombinant lines, hybrids and total genotypes. Cluster analyses were done for each level of genetic variation. The percentage of polymorphism varied between 34 and 54\% for AFLP, and between 40 and 78\% for polypeptide profiles. Higher polymorphism was observed in the group of hybrids. The genetic variability was $100 \%$ for titratable acidity and $34 \%$ for firmness, and the highest values were found in the parents. The genetic similarity varied among genotypes according to the level of genetic variation; however, the clustering consistency of some recombinant lines and their hybrids was conserved, which evidenced associations among molecular and phenotypic data.
\end{abstract}

Index terms: Lycopersicon, biotechnology, fruit quality, plant breeding, plant genetic resources.

\section{Introducción}

El tomate cultivado (Lycopersicon esculentum Mill., $2 \mathrm{n}=2 \mathrm{x}=24$ ) es una especie autógama en que la calidad de los frutos juega un rol muy importante. El género Lycopersicon incluye aproximadamente 18 especies, las cuales muestran un alto grado de homosecuencialidad en sus cromosomas (Peralta et al., 2008). Pratta et al. (1996) y Zorzoli et al. (1998) han demostrado que las especies silvestres constituyen una fuente de variabilidad genética para prolongar la vida poscosecha de los frutos. Zorzoli et al. (2000) comenzaron una selección antagónica-divergente, conducida según el método genealógico, en relación al peso y a la vida poscosecha de los frutos, en una generación $F_{2}$ proveniente de un cruzamiento entre una cultivar estándar de tomate y la accesión LA 722 de Lycopersicon pimpinellifolium (L.) Mill. Como resultado de seis ciclos de selección, se generaron 17 líneas recombinantes (RILs), divergentes en los valores de peso y vida poscosecha de los frutos (Rodríguez et al., 2006a, 2006b). 
Pratta (2004) utilizó marcadores moleculares de ADN de tipo AFLP ("amplified fragment length polymorphism") para caracterizar a los progenitores Caimanta y LA722, la generación $\mathrm{F}_{1}$ y las 17 RILs, y logró la identificación de marcadores moleculares asociados al peso, la forma, el contenido en sólidos solubles, la acidez, el color, la dureza y la vida poscosecha de los frutos. La mayor cantidad de asociaciones (detectadas por análisis de único punto) con fragmentos polimórficos correspondió al peso y a la vida en estantería, caracteres usados como criterios de selección.

La acumulación de pigmentos carotenoides y el ablandamiento del fruto permiten distinguir diversos estados en la madurez: verde maduro, rompiente, pintón, rosa, rojo claro y rojo maduro. A lo largo de este proceso se producen cambios en la expresión de proteínas, por lo que los perfiles polipeptídicos del fruto en distintos estados de madurez también constituyen marcadores moleculares para identificar genotipos. Rodríguez et al. (2008) caracterizaron la generación segregante derivada de un cruzamiento entre una cultivar homocigota para el gen mutante de la madurez (nor - non ripening) y una accesión de L. esculentum var. cerasiforme (Dunal) A. Garay, por los perfiles polipeptídicos del pericarpio, en tres estados de madurez. A partir de estos experimentos, se detectaron polipéptidos asociados a la duración de los diferentes estados de madurez, el peso y la vida poscosecha de los frutos.

El diseño de cruzamientos dialélicos es uno de los modelos genético-estadísticos más conocidos y uno de los que ofrece mayor información sobre la genética de los caracteres cuantitativos (Griffing, 1956). Se basa, por un lado, en la comparación entre un grupo de parentales y todas las combinaciones híbridas entre ellos, para determinar los valores de cruzamiento de los progenitores - conocidos como valores reproductivos, de mejora o "breeding values", estimados a través de modelos tipo I, de efectos fijos. Los modelos tipo II, de efectos aleatorios, permiten, por otro lado, conocer el tipo y la magnitud de la variación genética. A través del análisis dialélico, se puede también obtener una aproximación a la genética molecular subyacente a caracteres cuantitativos. Rebai \& Goffinet $(1993,2000)$ extendieron el uso del diseño de cruzamientos dialélicos y presentaron un modelo general basado en regresión para el mapeo de QTL (quantitative trait loci, o loci de caracteres cuantitativos). La presencia/ausencia de fragmentos polimórficos en parentales o híbridos, con valores discrepantes de aptitud combinatoria general (ACG) o específica (ACE) para un determinado carácter cuantitativo, indica asociaciones genéticas entre el marcador y el atributo fenotípico bajo análisis, lo que posibilita la detección de QTLs (Xie et al., 1998; $\mathrm{Xu}, 1998$; Jourjon et al., 2005).

El objetivo de este trabajo fue caracterizar, por marcadores moleculares y atributos cuantitativos de calidad del fruto de tomate, los genotipos de un diseño de cruzamientos dialélicos completo sin recíprocos, para estimar el tipo y la magnitud de la variación genética presente y detectar posibles asociaciones entre ambos conjuntos de datos.

\section{Materiales y Métodos}

Los experimentos se realizaron en el Laboratorio de Cultivo in vitro de Tejidos Vegetales y Biología Molecular, de la Facultad de Ciencias Agrarias de la Universidad Nacional de Rosario, ubicado en Zavalla,

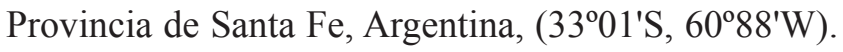
El material genético fue compuesto por cinco de las líneas recombinantes obtenidas por Rodríguez et al. (2006a), que fueron los parentales del dialélico: RIL1, RIL8, RIL9, RIL15 y RIL18. Se obtuvieron los híbridos entre ellas, siguiendo un diseño de cruzamiento dialélico completo sin recíprocos (Modelo I, Método 2). Quince plantas por genotipo fueron transplantadas para invernadero, en un diseño completamente aleatorizado; los resultados del análisis dialélico clásico de estos materiales fue reportado por Marchionni Basté et al. (2010).

Para la obtención de los perfiles de AFLP, la extracción de ADN genómico se realizó con un kit comercial a partir de hojas jóvenes, que fueron recolectadas en la cosecha 2007/2008 y conservadas a $-80^{\circ} \mathrm{C}$. Se realizaron tres extracciones independientes por genotipo (parentales e híbridos), para determinar la repetibilidad de los resultados. La cantidad y calidad del ADN extraído se evaluó en geles de agarosa al $1 \%$, por comparación con un estándar de ADN del bacteriófago lambda. Se digirieron 1,5 $\mu \mathrm{g}$ del ADN genómico con 10 unidades de EcoRI y 10 unidades de MseI en $40 \mu \mathrm{L}$ de solución, y las muestras fueron incubadas a $37^{\circ} \mathrm{C}$ durante dos horas. Los fragmentos resultantes de la digestión se ligaron a los adaptadores 
específicos de doble cadena GACTGCGTACCAATTC (adaptador EcoRI0, concentración final 0,025 $\mu \mathrm{mol} \mathrm{L}^{-1}$ ) y GATGAGTCCTGAGTAA (adaptador MseI0, concentración final $0,025 \mu \mathrm{mol} \mathrm{L} \mathrm{L}^{-1}$ ), por incubación a $37^{\circ} \mathrm{C}$ durante dos horas, con tres unidades de ligasa $\mathrm{T} 4$ en un volumen final de $10 \mu \mathrm{L}$. La solución de preamplificación se preparó con $1 \mu \mathrm{L}$ del ADN digerido/ligado, más $75 \mathrm{ng}$ de cada cebador $($ EcoRI +1 : EcoRI0A y MseI +1 : MseI0C), $1 \mu \mathrm{L}$ de dNTPs y $0,2 \mu \mathrm{L}$ de Taq-polimerasa, en un volumen final de $25 \mu \mathrm{L}$. Las condiciones de preamplificación fueron de 30 ciclos a cada $30 \mathrm{~s} \mathrm{a} 94^{\circ} \mathrm{C}, 1 \mathrm{~min}$ a $56^{\circ} \mathrm{C}$ y $1 \mathrm{~min}$ a $72^{\circ} \mathrm{C}$ por ciclo. Los fragmentos preamplificados fueron diluidos 1:10 (v:v).

La solución de amplificación se preparó con $3 \mu \mathrm{L}$ de la dilución de ADN preamplificado, más 75 ng de cada cebador selectivo (EcoRI +3 y MseI +3$), 1 \mu \mathrm{L}$ de dNTPs y $0,2 \mu \mathrm{L}$ de Taq-polimerasa, en un volumen final de $25 \mu \mathrm{L}$. Las secuencias de los cebadores utilizados en la amplificación, seleccionadas según los resultados de Pratta (2004), fueron: combinación 1 (CCS1, MseI31 + EcoRI44): MseI0CAC + EcoRI0ATC; combinación 2 (CCS2, MseI33 + EcoRI40): MseI0CAT + EcoRI0AGC; combinación 3 (CCS3, MseI33 + EcoRI46): MseI0CAT + EcoRI0ATT; combinación 4 (CCS4, MseI34 + EcoRI40): MseI0CTA + EcoRI0AGC; y combinación 5 (CCS5, (MseI34 + EcoRI46): MseI0CTA) + EcoRI0ATT.

Las condiciones de amplificación fueron, en este caso, de tipo "touch down", y comenzaron con un ciclo de $30 \mathrm{~s}$ a $94^{\circ} \mathrm{C}, 30 \mathrm{~s}$ a $65^{\circ} \mathrm{C}$ y $1 \mathrm{~min}$ a $72^{\circ} \mathrm{C}$. En los 10 ciclos sucesivos, la temperatura de annealing fue disminuida $1{ }^{\circ} \mathrm{C}$ por ciclo, hasta llegar a $56^{\circ} \mathrm{C}$. A partir de entonces, se repitieron 23 ciclos de $30 \mathrm{~s} \mathrm{a} 94^{\circ} \mathrm{C}, 1 \mathrm{~min}$ a $56^{\circ} \mathrm{Cy} 1 \mathrm{~min}$ a $72^{\circ} \mathrm{C}$. En todas las reacciones, se emplearon los buffers comerciales de cada enzima. Los fragmentos amplificados se separaron en geles de poliacrilamida al $6 \%$, en condiciones desnaturalizantes $\left(7,5 \mathrm{~mol} \mathrm{~L}^{-1}\right.$ urea y $1 \mathrm{X}$ TBE). En cada corrida, se agregaron marcadores comerciales de peso molecular (CienMarker, Biodynamics SRL, Buenos Aires, Argentina). Para el revelado, se utilizó la técnica no radioactiva de nitrato de plata.

Para la obtención de los perfiles de polipéptidos, en todos los genotipos, se cosecharon frutos en los estados de madurez verde maduro (VM), pintón (P), rojo maduro alcanzado en planta (RMP) y rojo maduro alcanzado en estantería (RME). Los frutos fueron conservados a $-80^{\circ} \mathrm{C}$, y la extracción de proteínas se realizó siguiendo el protocolo de Rodríguez et al. (2008). Para determinar la repetibilidad de los resultados, se evaluaron dos muestras independientes por genotipo y por estado de madurez. El pericarpio se homogeneizó en $\mathrm{N}_{2}$ líquido con mortero, en presencia de $1,5 \mathrm{~mL}$ de buffer de extracción (100 $\mathrm{mmol} \mathrm{L}^{-1}$ Tris/ $\mathrm{HCl} \mathrm{pH} 8,0,1 \mathrm{mmol} \mathrm{L}^{-1}$ EDTA, 1 mmol L-1 PMSF, $2 \%$ de $\beta$-mercaptoetanol) y $6 \mathrm{~mL}$ de fenol saturado con Tris buffer $100 \mathrm{mmol} \mathrm{L}^{-1}$ (pH 8,0). Se centrifugó a $4.200 \mathrm{rpm}$ durante $10 \mathrm{~min}$ a $4^{\circ} \mathrm{C}$, se removió la fase fenólica, se extrajo nuevamente con un volumen de buffer de extracción, y se mezcló con cuatro volúmenes de acetato de amonio en metanol $\left(0,1 \mathrm{~mol} \mathrm{~L}^{-1}\right)$. Las muestras se incubaron a $-20^{\circ} \mathrm{C}$ durante la noche. Los polipéptidos se precipitaron por centrifugación a $4.200 \mathrm{rpm}$ durante $15 \mathrm{~min}$ a $4^{\circ} \mathrm{C}$. El pellet se lavó dos veces con acetato de amonio en metanol $\left(0,1 \mathrm{~mol} \mathrm{~L}^{-1}\right)$ y una vez con acetona al $80 \%$, y se resuspendió en buffer de siembra $\left(25 \mathrm{mmol} \mathrm{L}^{-1}\right.$ Tris $\mathrm{pH}$ 6,8, $1 \%$ SDS, $10 \%$ glicerol, $5 \%$ de $\beta$-mercaptoetanol y $0,002 \%$ de bromofenol blue). Los polipéptidos se separaron con un gel de poliacrilamida al $10 \%$ en condiciones desnaturalizantes, sembrando $30 \mu \mathrm{g}$ en cada calle, cuantificados por la técnica de Bradford. La electroforesis se realizó a potencia constante $(35 \mathrm{~mA})$ $\mathrm{y}$, para la tinción, se empleó la técnica de Coomasie Brillant Blue.

Para la caracterización fenotípica, se cosechó un número mínimo de diez tomates por planta de las cinco RILs y los 10 híbridos, para evaluar los siguientes caracteres cuantitativos: diámetro (D), en $\mathrm{cm}$; altura (A), en cm; forma (Fo), cociente altura/diámetro; peso (P), g; vida poscosecha (VP), medida por el número de días transcurridos de la cosecha al estado pintón, y el primer síntoma de deterioro de los frutos almacenados a $25 \pm 3^{\circ} \mathrm{C}$, según Schuelter et al. (2002); color (a/b), cociente entre absorbencia a longitud de onda de $540 \mathrm{~nm}$ y absorbencia a longitud de onda de $675 \mathrm{~nm}$, medidas con un cromómetro estándar CR400, (Konica Minolta, Tokyo, Japón); firmeza (F), determinada con un tester de presión de fruto 12.5 N tipo Durofel DFT 100 (Agro-Technologie, Forges Les Eaux, Francia), con una punta de $0,10 \mathrm{~cm}^{2}$ en una escala de 0-100; contenido en sólidos solubles (SS), en ${ }^{\circ}$ Brix, determinado con un refractómetro de mano PCE010 (PCE Instruments Ibérica S.L., Tobarra, España), en el jugo homogeneizado de tejido del pericarpio; $\mathrm{pH}$; y acidez titulable (AT), en gramos de ácido cítrico $100 \mathrm{~g}^{-1}$ de jugo homogeneizado. 
Los análisis estadísticos, tanto para los perfiles de AFLP como para los de polipéptidos, consistieron en analizar la presencia o ausencia de bandas repetibles y en cuantificar el número total de bandas y el porcentaje de bandas polimórficas entre los diferentes genotipos, por combinación de cebador selectivo y por estado de madurez, respectivamente. Cada tipo de marcador se analizó en el grupo de parentales y en el de híbridos en forma independiente, y luego se compararon ambos grupos, relevando en el de híbridos el número de bandas con comportamiento de novo: bandas ausentes en ambos progenitores pero detectadas en el híbrido respectivo, o bien, bandas presentes en ambos progenitores pero no detectadas en el híbrido respectivo. Luego se calcularon las distancias de Jaccard entre todos los genotipos con los datos de marcadores de AFLP y de perfiles polipeptídicos por separado, y se aplicó un análisis de cluster jerárquico para su agrupamiento a cada conjunto de datos moleculares.

Para los caracteres cuantitativos, los genotipos se compararon por ANOVA a un criterio de clasificación. A partir de los cuadrados medios, se estimó la variancia genética y se calculó el porcentaje de variabilidad genética sobre el total de variabilidad fenotípica en el grupo de RILs, en el de híbridos y en total (Kearsey \& Pooni, 1996). El conjunto de genotipos se agrupó luego según la media de los caracteres cuantitativos, con los que se calculó la distancia euclidiana entre ellos.

Dado que cada una de las caracterizaciones realizadas (marcador de AFLP, marcador de polipéptidos y caracteres cuantitativos) refleja un nivel de variación genética diferente, se llevó a cabo también el agrupamiento de los parentales e híbridos, según el total de los datos moleculares y fenotípicos, por el cálculo de las distancias de Gower entre los genotipos (Gower, 1971). En todos los casos, se empleó el método UPGMA ("unweighted pairgroupmethod with arithmetic mean"), también llamado promedio (o "average linkage"), para la construcción de los dendrogramas. Finalmente, se analizó el comportamiento de las bandas moleculares polimórficas en parentales e híbridos, en relación a los valores de ACG y ACE de todos los caracteres cuantitativos, para detectar asociaciones entre ambos conjuntos de datos que sugerirían la presencia de QTLs para los atributos fenotípicos analizados, marcados por los fragmentos de ADN y los polipéptidos.

\section{Resultados y Discusión}

En el Cuadro 1, se presentan los resultados de la caracterización molecular. En el caso de los marcadores de AFLP, el polimorfismo para los genotipos del cruzamiento dialélico de RILs e híbridos varió en $21 \%$ entre las combinaciones que presentaron los valores extremos. Al considerar el total de las combinaciones de cebadores, el polimorfismo, semejante en los grupos de RILs y de híbridos, fue 5,1 y 3,8\% superior para el conjunto de todas las combinaciones $y$ genotipos respecto a estos valores. Para los perfiles de polipéptidos, el porcentaje de polimorfismo varió

Cuadro 1. Caracterización molecular de los padres (RILs) e híbridos involucrados en el diseño de cruzamientos dialélicos de tomate.

\begin{tabular}{|c|c|c|c|c|c|c|c|c|c|c|c|}
\hline \multirow[t]{2}{*}{ Característica } & \multicolumn{6}{|c|}{ Perfiles de AFLP(1) } & \multicolumn{5}{|c|}{ Perfiles de polipéptidos totales del pericarpio } \\
\hline & CCS1 & CCS2 & CCS3 & CCS4 & CCS5 & Total & VM & $\mathrm{P}$ & RMP & RME & Total \\
\hline & \multicolumn{11}{|c|}{ RILs } \\
\hline Bandas totales & 106 & 77 & 118 & 92 & 130 & 523 & 18 & 17 & 15 & 17 & 67 \\
\hline Bandas polimórficas & 44 & 32 & 40 & 45 & 51 & 212 & 11 & 7 & 6 & 9 & 33 \\
\hline \multirow[t]{2}{*}{ Porcentaje de polimorfismo } & 41,5 & 41,6 & 33,9 & 48,9 & 39,2 & 40,5 & 61,1 & 41,2 & 40,0 & 52,9 & 49,3 \\
\hline & \multicolumn{11}{|c|}{ Híbridos } \\
\hline Bandas totales & 105 & 79 & 118 & 98 & 129 & 529 & 18 & 19 & 18 & 17 & 72 \\
\hline Bandas de novo & 3 & 5 & 3 & 13 & 8 & 32 & 3 & 3 & 7 & 3 & 16 \\
\hline Bandas polimórficas & 38 & 34 & 42 & 53 & 54 & 221 & 14 & 10 & 8 & 7 & 39 \\
\hline \multirow[t]{2}{*}{ Porcentaje de polimorfismo } & 36,2 & 43,0 & 35,6 & 54,1 & 41,9 & 41,8 & 77,8 & 52,6 & 44,4 & 41,2 & 54,2 \\
\hline & \multicolumn{11}{|c|}{ RILs + Híbridos } \\
\hline Bandas totales & 107 & 79 & 119 & 98 & 130 & 533 & 18 & 19 & 18 & 18 & 73 \\
\hline Bandas polimórficas & 48 & 37 & 43 & 56 & 59 & 243 & 14 & 10 & 11 & 10 & 45 \\
\hline Porcentaje de polimorfismo & 44,9 & 46,8 & 36,1 & 57,1 & 45,4 & 45,6 & 77,8 & 52,6 & 61,1 & 55,6 & 61,6 \\
\hline
\end{tabular}

${ }^{(1)}$ Combinaciones de cebadores selectivos (CCS): 1, MseI31 + EcoRI44; 2, MseI33 + EcoRI40; 3: MseI33 + EcoRI46; 4, MseI34 + EcoRI40; 5, MseI34 + EcoRI46. VM, frutos en el estado verde maduro; P, frutos en el estado pintón; RMP, frutos en el estado rojo maduro alcanzado en planta; RME, frutos en el estado rojo maduro alcanzado en estantería. 
25,2\% según el estado de madurez. No se encontró una asociación entre estado de madurez y polimorfismo en perfiles polipeptídicos, aunque, como se destaca más abajo, el mayor polimorfismo haya correspondido al estado verde maduro. Considerándose todos los estados, el grupo de RILs presentó un porcentaje de bandas polimórficas menor que el de híbridos. El porcentaje de polimorfismo general fue de 12,3 y $7,4 \%$ superior a esos valores, respectivamente. Respecto a las bandas con comportamiento de novo, si bien el número absoluto fue mayor para los marcadores de ADN, el porcentaje fue mayor para los perfiles polipeptídicos $(21,9 \%$ para los polipéptidos versus $6,0 \%$ para AFLP).

Los valores medios, errores estándares y los porcentajes de variabilidad genética (PVG) de los caracteres cuantitativos de calidad de fruto se presentan en el Cuadro 2. Los valores de PVG variaron entre $34 \%$ para firmeza y $100 \%$ para acidez en el conjunto de genotipos, y fueron, con excepción de la firmeza, mayores en el grupo de RILs que en el de híbridos.

Los resultados del análisis de cluster jerárquico, con cada nivel de variación genética analizado y con el total de los datos, se presentan en la Figura 1. Se observa que los genotipos fueron distintos según el nivel de variación genética empleado como criterio para el agrupamiento. Sin embargo, el grupo formado por algunas RILs (15 y 18) y la mayoría de sus híbridos fue constante en los tres dendrogramas correspondientes a cada nivel de variación analizada. Además, y a modo de ejemplo de asociación entre datos moleculares y caracteres fenotípicos, la RIL 15 se destacó por sus valores negativos de ACG y ACE para VP; dos de sus híbridos (1x15 y 15x9) tuvieron valores positivos de ACE y los otros dos (15x8 y 18x15), valores negativos (Marchionni Basté et al., 2010). En concordancia, la RIL15 presentó una banda polipeptídica de $45 \mathrm{kDa}$ en el estado VM, que estuvo presente en los híbridos $15 \times 8$ y $18 \mathrm{x} 15$, pero ausente en $1 \mathrm{x} 15$ y $15 \mathrm{x} 9$.

En este experimento, se detectó la existencia de variabilidad en diferentes niveles del flujo de la información genética: ADN, polipéptidos y atributos fenotípicos, en un grupo de RILs y sus híbridos obtenidos según un diseño dialélico. Sin embargo, la variabilidad encontrada con los marcadores de AFLP, los perfiles polipeptídicos y los caracteres cuantitativos relacionados a la calidad de frutos fue distinta en calidad y magnitud.

Cuadro 2. Valor medio \pm error estándar y porcentaje de variabilidad genética $\left(\mathrm{PVG}_{\mathrm{P}}\right.$, parental; $\mathrm{PVG}_{\mathrm{H}}$, híbridos; $\mathrm{PVG}$, total de genotipos) de caracteres cuantitativos de calidad de fruto, en cruzamientos dialélicos de tomate.

\begin{tabular}{|c|c|c|c|c|c|c|c|c|c|c|}
\hline \multirow[t]{2}{*}{ Genotipo } & \multicolumn{10}{|c|}{ Caracteres cuantitativos $^{(1)}$} \\
\hline & $\begin{array}{l}\text { Diámetro } \\
(\mathrm{cm})\end{array}$ & $\begin{array}{l}\text { Altura } \\
(\mathrm{cm})\end{array}$ & Forma & $\begin{array}{c}\text { Peso } \\
\text { (g) }\end{array}$ & $\begin{array}{c}\text { Vida poscosecha } \\
\text { (días) }\end{array}$ & Color & Firmeza & $\begin{array}{c}\text { Sólidos solubles } \\
\left({ }^{\circ} \text { Brix }\right)\end{array}$ & $\mathrm{pH}$ & $\begin{array}{l}\text { Acidez } \\
\text { titulable }\end{array}$ \\
\hline & \multicolumn{10}{|c|}{ Parental } \\
\hline RIL1 & $3,55 \pm 0,06$ & $2,85 \pm 0,09$ & $0,81 \pm 0,02$ & $21,66 \pm 1,35$ & $24,79 \pm 1,18$ & $1,04 \pm 0,15$ & $54,17 \pm 7,06$ & $5,54 \pm 0,25$ & $4,96 \pm 0,05$ & $0,32 \pm 0,02$ \\
\hline RIL8 & $1,58 \pm 0,06$ & $1,42 \pm 0,04$ & $0,90 \pm 0,01$ & $2,34 \pm 0,21$ & $17,98 \pm 0,62$ & $1,14 \pm 0,11$ & $54,55 \pm 4,68$ & $9,30 \pm 0,66$ & $4,51 \pm 0,09$ & $0,65 \pm 0,02$ \\
\hline RIL9 & $1,83 \pm 0,06$ & $1,63 \pm 0,04$ & $0,89 \pm 0,01$ & $3,81 \pm 0,32$ & $18,70 \pm 0,43$ & $1,37 \pm 0,05$ & $53,39 \pm 3,38$ & $7,04 \pm 0,18$ & $4,35 \pm 0,05$ & $0,79 \pm 0,04$ \\
\hline RIL15 & $2,84 \pm 0,09$ & $2,22 \pm 0,07$ & $0,79 \pm 0,02$ & $11,59 \pm 1,04$ & $14,41 \pm 1,49$ & $1,12 \pm 0,16$ & $54,32 \pm 4,52$ & $7,91 \pm 0,34$ & $4,61 \pm 0,11$ & $0,44 \pm 0,03$ \\
\hline RIL18 & $2,83 \pm 0,06$ & $2,77 \pm 0,07$ & $0,97 \pm 0,02$ & $13,72 \pm 0,72$ & $21,11 \pm 1,20$ & $1,15 \pm 0,10$ & $52,47 \pm 4,51$ & $7,69 \pm 0,26$ & $4,72 \pm 0,07$ & $0,37 \pm 0,03$ \\
\hline \multirow[t]{2}{*}{$\mathrm{PVG}_{\mathrm{P}}(\%)$} & 91 & 92 & 73 & 92 & 55 & 84 & 0 & 91 & 83 & 100 \\
\hline & \multicolumn{10}{|c|}{ Híbrido } \\
\hline$(1 \times 8)$ & $2,21 \pm 0,05$ & $1,90 \pm 0,03$ & $0,88 \pm 0,01$ & $5,80 \pm 0,23$ & $26,51 \pm 1,24$ & $1,18 \pm 0,06$ & $47,15 \pm 4,68$ & $8,26 \pm 0,24$ & $4,50 \pm 0,07$ & $0,59 \pm 0,05$ \\
\hline$(1 \times 9)$ & $2,17 \pm 0,03$ & $2,02 \pm 0,02$ & $0,94 \pm 0,02$ & $5,81 \pm 0,02$ & $24,60 \pm 1,27$ & $1,19 \pm 0,07$ & $55,71 \pm 6,88$ & $7,09 \pm 0,21$ & $4,65 \pm 0,11$ & $0,39 \pm 0,05$ \\
\hline$(1 \times 15)$ & $2,40 \pm 0,07$ & $2,09 \pm 0,07$ & $0,89 \pm 0,01$ & $7,31 \pm 0,05$ & $21,93 \pm 1,40$ & $1,25 \pm 0,09$ & $45,53 \pm 7,40$ & $5,49 \pm 0,44$ & $4,49 \pm 0,09$ & $0,42 \pm 0,06$ \\
\hline$(18 \times 1)$ & $2,59 \pm 0,11$ & $2,59 \pm 0,09$ & $1,02 \pm 0,02$ & $10,62 \pm 0,99$ & $23,08 \pm 1,80$ & $1,15 \pm 0,12$ & $54,85 \pm 7,72$ & $6,48 \pm 0,34$ & $4,54 \pm 0,08$ & $0,44 \pm 0,07$ \\
\hline$(9 \times 8)$ & $1,80 \pm 0,04$ & $1,67 \pm 0,07$ & $0,93 \pm 0,02$ & $3,36 \pm 0,26$ & $18,77 \pm 0,93$ & $1,27 \pm 0,08$ & $48,89 \pm 6,27$ & $8,09 \pm 0,87$ & $4,42 \pm 0,08$ & $0,55 \pm 0,08$ \\
\hline$(15 \times 8)$ & $1,84 \pm 0,04$ & $1,64 \pm 0,04$ & $0,89 \pm 0,01$ & $3,50 \pm 0,22$ & $15,47 \pm 0,75$ & $1,39 \pm 0,07$ & $52,39 \pm 5,12$ & $6,89 \pm 0,36$ & $4,62 \pm 0,11$ & $0,55 \pm 0,04$ \\
\hline$(18 \times 8)$ & $2,57 \pm 0,05$ & $2,47 \pm 0,06$ & $0,97 \pm 0,02$ & $10,42 \pm 0,66$ & $20,58 \pm 1,06$ & $1,23 \pm 0,11$ & $44,87 \pm 7,07$ & $7,31 \pm 0,27$ & $4,74 \pm 0,10$ & $0,33 \pm 0,02$ \\
\hline$(15 \times 9)$ & $2,36 \pm 0,04$ & $2,11 \pm 0,03$ & $0,91 \pm 0,01$ & $7,37 \pm 0,32$ & $21,42 \pm 0,84$ & $1,24 \pm 0,12$ & $43,31 \pm 8,33$ & $7,20 \pm 0,22$ & $4,50 \pm 0,09$ & $0,55 \pm 0,04$ \\
\hline$(18 \times 9)$ & $1,96 \pm 0,05$ & $1,89 \pm 0,05$ & $0,98 \pm 0,01$ & $4,62 \pm 0,31$ & $20,67 \pm 0,98$ & $1,26 \pm 0,10$ & $49,00 \pm 7,13$ & $7,23 \pm 1,52$ & $4,62 \pm 0,08$ & $0,40 \pm 0,07$ \\
\hline$(18 \times 15)$ & $2,63 \pm 0,08$ & $2,41 \pm 0,09$ & $0,92 \pm 0,03$ & $10,64 \pm 0,95$ & $14,68 \pm 0,88$ & $1,19 \pm 0,17$ & $43,86 \pm 8,44$ & $7,35 \pm 0,53$ & $4,65 \pm 0,18$ & $0,46 \pm 0,03$ \\
\hline $\mathrm{PVG}_{\mathrm{H}}(\%)$ & 72 & 71 & 42 & 73 & 54 & 35 & 34 & 62 & 43 & 70 \\
\hline $\mathrm{PVG}_{\mathrm{T}}(\%)$ & 91 & 84 & 63 & 82 & 55 & 46 & 34 & 71 & 83 & 100 \\
\hline
\end{tabular}

${ }^{(1)}$ Caracteres cuantitativos: forma, cociente altura/diámetro; color, cociente entre absorbencia a longitud de onda de $540 \mathrm{~nm} / \mathrm{absorbencia} \mathrm{a} 675 \mathrm{~nm}$; firmeza, escala de $0-100$; acidez titulable, gramos de ácido cítrico $100 \mathrm{~g}^{-1}$ de jugo homogeneizado. 
En el nivel molecular, los perfiles polipeptídicos presentaron la mayor variabilidad entre genotipos, que alcanzó un máximo de $77,8 \%$ en el estado verde maduro. Este alto valor de polimorfismo se asoció con la presencia de un número mayor de bandas de novo. El mayor porcentaje de polimorfismo y de bandas de novo, para polipéptidos del pericarpio en el estado verde maduro, también fue encontrado por Rodríguez et al. (2008), lo que sugiere una mayor conservación entre genotipos de los productos de la expresión génica en estados más avanzados de la madurez, debido, posiblemente, a funciones fisiológicas específicas para el desarrollo del fruto. Por este motivo, el polimorfismo para los perfiles polipeptídicos en los estados pintón, rojo maduro en planta y rojo maduro en estantería es menor que en el estado verde maduro, en el cuál se observa la mayor variabilidad entre los genotipos. Además,

A
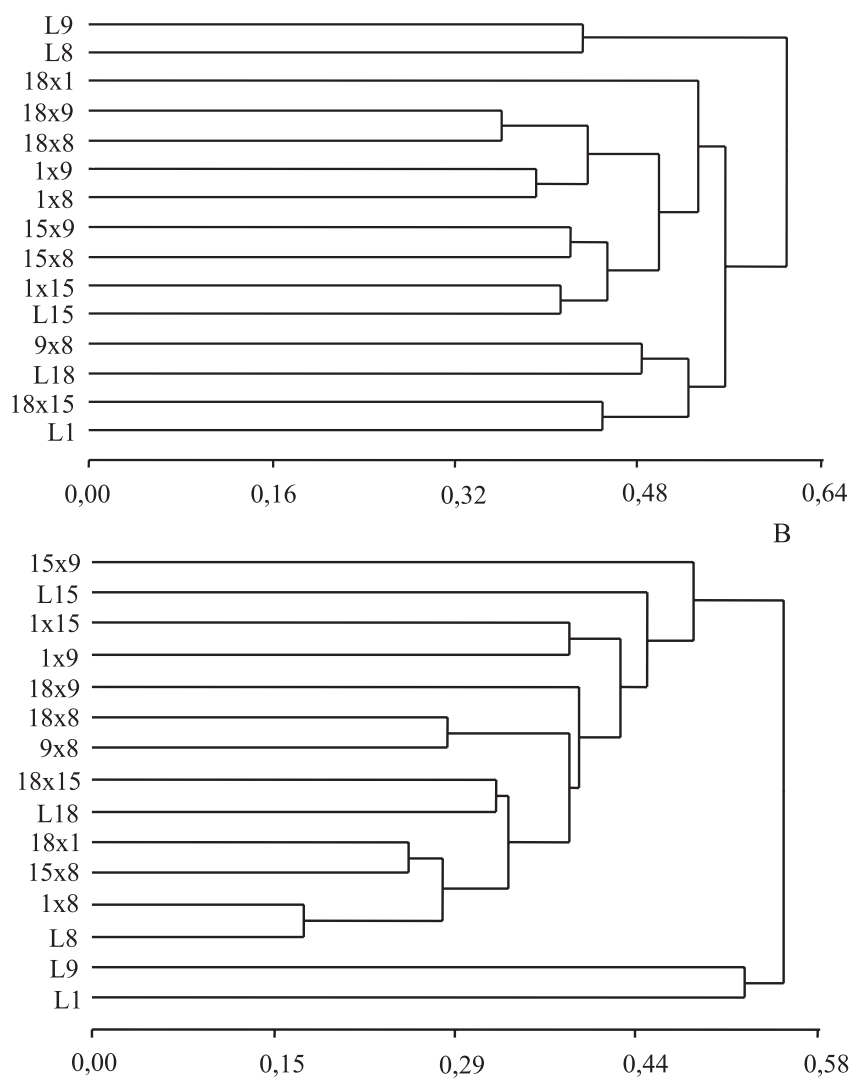

los comportamientos de novo en polipéptidos podrían deberse a reorganizaciones cromosómicas, que se dan frecuentemente en cruzamientos interespecíficos, como el que originó las RILs empleadas en este trabajo, y que ayudan a superar el estrés genómico que se produce al combinarse dos entidades genéticas evolutivamente alejadas, o bien a interacciones intra e interloci entre los genomas de los progenitores (Rieseberg, 2001).

Las diferentes combinaciones de cebadores selectivos que se usaron para generar los AFLP mostraron un importante grado de variabilidad entre ellas, y fue mayor el porcentaje de polimorfismo en los perfiles obtenidos por CCS4 (48,9\% para las RILs y $54,1 \%$ para los híbridos). Esta variabilidad dentro y entre diversas combinaciones fue también demostrada por Pratta (2004). Tal como es lo esperado para este tipo de marcadores de ADN que se comportan como dominantes, no fueron encontradas grandes diferencias

$\mathrm{C}$
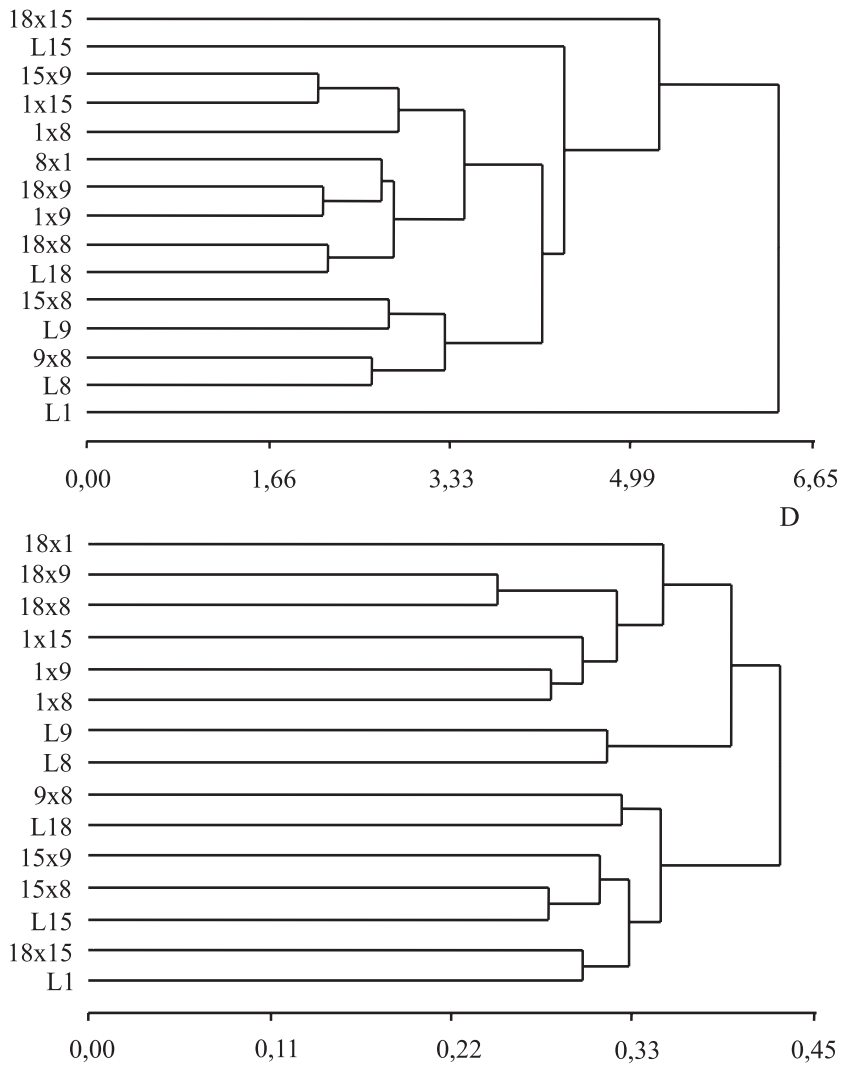

Figura 1. Análisis de agrupamiento de los genotipos de tomate (RILs, representadas por L, e híbridos, sólo por números), involucrados en el diseño de cruzamientos dialélicos con cada nivel de variación genética analizado y con el total de los datos. Agrupamientos: A, por AFLP, con cinco combinaciones de cebadores selectivos; B, por perfiles polipeptídicos, en cuatro estados de madurez; C, caracteres cuantitativos de calidad del fruto; D, por el total de los datos. A y B, distancia de Jaccard 1-S; C, distancia euclidiana; D, distancia de Gower 1-S. 
en la variabilidad de los grupos de RILs e híbridos. El menor porcentaje de bandas de novo, detectadas por AFLP respecto a polipéptidos, indicaría que la estructura genómica se conserva en los cruces entre RILs. Por lo tanto, las bandas de novo, observadas en los perfiles proteicos, deberían tener su origen principalmente en interacciones intra e interloci que suceden en los híbridos y afectan la expresión génica, tal como propuesto por Rodríguez et al. (2008).

Los diferentes valores de PVG para los caracteres estudiados indican la presencia de una mayor variabilidad fenotípica debida a variaciones genéticas entre las RILs, mientras que los híbridos presentaron una mayor similitud entre sí. Esto podría ser, posiblemente, debido a que en las $F_{1}$ s se reestablecen combinaciones similares de alelos al estado heterocigoto, puesto que en cada RIL se han dispersado los alelos presentes en los parentales de la cultivar Caimanta y LA722. De hecho, los híbridos entre RILs son muy similares a la $\mathrm{F}_{1}$ (cv. Caimanta x LA722).

Observándose los diferentes clusters jerárquicos de la Figura 1, se desprende que el agrupamiento con los perfiles de AFLP se presentó como el más representativo de la variabilidad en comparación con el agrupamiento obtenido para el conjunto de los datos. Sin embargo, esto podría deberse a que es el nivel de variación genética que más variables aportó al conjunto de datos, por la gran cantidad de bandas generadas por esta técnica molecular. En términos generales, los análisis de agrupamiento, según los diferentes niveles de variación genética, reflejaron la diferencia en la calidad y magnitud de variabilidad detectada por cada conjunto de datos. Sin embargo, existiría una asociación entre los perfiles de AFLP, los polipeptídicos y los caracteres cuantitativos en este grupo de genotipos, como queda demostrado por la constancia en el agrupamiento de algunas RILs y sus híbridos y la correspondencia entre el polimorfismo para los marcadores moleculares y los valores de ACG y ACE para algunos caracteres. En este sentido, la similaridad encontrada en los agrupamientos de la línea 15 y sus híbridos, en todos los clusters realizados, indica una congruencia entre los valores que presentan estos genotipos para las características dicotómicas presencia/ausencia de fragmentos AFLP y polipéptidos y las características cuantitativas de atributos del fruto (Gower, 1971). Estas congruencias representan asociaciones entre los conjuntos de datos, lo que indica que los polimorfismos moleculares se corresponden con la variabilidad fenotípica, convirtiéndose en posibles marcadores de QTLs para los caracteres cuantitativos bajo estudio. Al respecto y a modo de ejemplo de lo propuesto, Rodríguez et al. (2008) informaron que un polipéptido de $47 \mathrm{kDa}$, expresado en el estado pintón, estuvo asociado a la vida poscosecha de los frutos en la generación $F_{2}$ de un cruzamiento entre una cultivar homocigota para el mutante de madurez nor y una accesión de L. esculentum var. cerasiforme. La masa molecular de ese polipéptido es muy similar a la del detectado en el estado verde maduro en este experimento (45 kDa), cuyo polimorfismo se corresponde con las ACG y ACE para la vida poscosecha de la RIL 15 y sus híbridos. En consecuencia, la estrategia de abordar el estudio de la genética molecular de caracteres cuantitativos, a través de un diseño de cruzamientos dialélicos, se convierte en una aproximación válida para detectar posibles QTLs de interés agronómico, que deberán validarse posteriormente en generaciones segregantes de los híbridos evaluados.

\section{Conclusiones}

1. La caracterización de genotipos que conforman un diseño de cruzamientos dialélicos completo sin recíprocos, por marcadores moleculares y atributos fenotípicos, permite avanzar en el conocimiento de la genética molecular de caracteres cuantitativos relacionados a la calidad de los frutos de tomate.

2. Hay diferencias en la calidad y magnitud de cada nivel de variación genética.

3. Existen asociaciones entre el polimorfismo en el nivel molecular y los valores de aptitud combinatoria general y específica de los caracteres cuantitativos.

\section{Agradecimientos}

Al Consejo Nacional de Investigaciones Científicas y Técnicas y a la Agencia Nacional de Promoción Científica y Tecnológica, por soporte financiero.

\section{Referencias}

GOWER, J.C. A general coefficient of similarity and some of its properties. Biometrics, v.27, p.857-871, 1971.

Pesq. agropec. bras., Brasília, v.46, n.5, p.509-516, maio 2011 
GRIFFING, B. Concept of general and specific combining ability in relation to diallel crossing systems. Australian Journal of Biological Sciences, v.9, p.463-493, 1956.

JOURJON, M.F.; JASSON, S.; MARCEL, J.; NGOM, B.; MANGIN, B. MCQTL: multi-allelic QTL mapping in multi-cross design. Bioinformatics v.21, p.128-130, 2005.

KEARSEY, M.J.; POONI, H.S. The genetical analysis of quantitative traits. London: Chapman and Hall, 1996. 382p.

MARCHIONNI BASTÉ, E.; LIBERATTI, D.R.; MAHUAD, S.L.; RODRÍGUEZ, G.R.; PRATTA, G.R.; ZORZOLI, R.; PICARDI, L.A. Diallel analysis for fruit traits among tomato recombinant inbred lines derived from an interspecific cross Solanum lycopersicum x S. pimpinellifolium. Journal of Applied Horticulture, v.12, p.1-25, 2010.

PERALTA, I.E.; SPOONER, D.M.; KNAPP, S. Taxonomy of wild tomatoes and their relatives (Solanum sect. Lycopersicoides, sect. Juglandifolia, sect. Lycopersicon: Solanaceae). Ann Arbor: American Society of Plant Taxonomists, 2008. 186p. (ASPT. Systematic botany monographs, 85).

PRATTA, G.R. Caracterización de genotipos discrepantes para el tiempo de madurez de los frutos en Lycopersicon. 2004. 120p. Tesis (Ph.D.) - Universidad Nacional de Rosario, Argentina.

PRATTA, G.R.; ZORZOLI, R.; PICARDI, L.A. Evaluación de caracteres de interés agronómico en especies del género Lycopersicon. Horticultura Argentina, v.15, p.25-32, 1996.

REBAI, A.; GOFFINET, B. More about quantitative trait locus mapping with diallel designs. Genetical Research, v.75, p.243-247, 2000 .

REBAI, A.; GOFFINET, B. Power of tests for QTL detection using replicated progenies derived from a diallel cross. Theoretical and Applied Genetics, v.86, p.1014-1022, 1993.
RIESEBERG, L.H. Polyploid evolution: keeping the peace at genomic reunions. Current Biology, v.11, p.925-928, 2001.

RODRÍGUEZ, G.R.; PRATTA, G.R.; ZORZOLI, R.; PICARDI, L.A. Evaluation of plant and fruit traits in recombinant inbred lines of tomato obtained from a cross between Lycopersicon esculentum and L. pimpinellifolium. Ciencia e Investigación Agraria, v.33, p.111-118, 2006a.

RODRÍGUEZ， G.R.; PRATTA， G.R.; ZORZOLI， R.; PICARDI, L.A. Recombinant lines obtained from an interspecific: cross between Lycopersicon species selected by fruit weight and fruit shelf life. Journal of the American Society for Horticultural Science, v.131, p.651-656, 2006b.

RODRÍGUEZ, G.R.; SEQUIN, L.; PRATTA, G.R.; ZORZOLI, R.; PICARDI, L.A. Protein profiling in $F_{1}$ and $F_{2}$ generations of two tomato genotypes differing in ripening time. Biologia Plantarum, v.52, p.548-552, 2008.

SCHUELTER, A.R.; FINGER, F.L.; CASALI, V.W.D.; BROMMONSCHENKEL, S.H.; OTONI, W.C. Inheritance and genetic linkage analysis of a firm-ripening tomato mutant. Plant Breeding, v.121, p.338-342, 2002.

XIE, C.; GESSLER, D.G.; XU, S. Combining different line crosses for mapping quantitative trait loci using the identical by descent-based variance component method. Genetics, v.149, p.1139-1146, 1998.

XU, S.Z. Mapping quantitative trait loci using multiple families of line crosses. Genetics, v.148, p.517-524, 1998.

ZORZOLI, R.; PRATTA, G.R.; PICARDI, L.A. Efecto de los mutantes nor y rin y de genes silvestres sobre características del fruto en Lycopersicon. Mendeliana, v.13, p.12-19, 1998.

ZORZOLI, R.; PRATTA, G.R.; PICARDI, L.A. Variabilidad genética para la vida postcosecha y el peso de los frutos en tomate en familias $\mathrm{F}_{3}$ de un híbrido interespecífico. Pesquisa Agropecuária Brasileira, v.35, p.2423-2427, 2000.

Recebido el 31 de deciembre de 2010 y aprobado el 3 de marzo de 2011

Pesq. agropec. bras., Brasília, v.46, n.5, p.509-516, maio 2011 